\title{
EFFECTS OF FERTILIZATION AND STRAW INCORPORATION ON BACTERIAL COMMUNITIES IN BLACK SOIL, NORTHEASTERN CHINA
}

\author{
ZENG, X. N. ${ }^{1}-$ SONG, Q. L. ${ }^{1}-$ YU, S. ${ }^{1}-$ ZHOU, M. ${ }^{2}-$ FENG, Y. J. ${ }^{1}-$ LAI, Y. C. ${ }^{3}-$ WANG, Q. ${ }^{1 *}$ \\ ${ }^{1}$ Institute of Crop Cultivation and Tillage, Heilongjiang Academy of Agricultural Sciences, \\ Harbin 150080, Heilongjiang Province, China \\ ${ }^{2}$ Department of Biological Center, Harbin Academy of Agricultural Sciences, Harbin 150070, \\ Heilongjiang Province, China \\ ${ }^{3}$ Heilongjiang Academy of Agricultural Sciences, Harbin 150080, Heilongjiang Province, China \\ "Corresponding author \\ e-mail: neauwq@163.com; phone: +86-0451-8666-8732; fax: +86-0451-8666-8732
}

(Received 20 $0^{\text {th }}$ Apr 2020; accepted $13^{\text {th }}$ Aug 2020)

\begin{abstract}
The effects of three treatments including no fertilizer or straw returned to the field (CK), chemical fertilizer with straw not returned to the field $(\mathrm{F})$ and no fertilizer with straw returned to the field (W) on the composition, diversity and structure of the soil bacterial community were examined. The results showed that a total of 1,833,232 reads and 25,843 operational taxonomic units (OTUs) were obtained through sequencing. The dominant bacteria of the black soil in the northeast $(>10 \%)$ were Proteobacteria, Acidobacteria, Bacteroidetes, Chloroflexi and actinomycetes. The soil bacterial diversity was greatly improved, but fertilization had little effect on the soil bacterial diversity. A stratified clustering map showed that the soil bacterial community structures after $\mathrm{W}$ and $\mathrm{F}$ treatments were similar to one another at genus level, and the soil bacterial community structures after treatment with $\mathrm{W}$ and $\mathrm{F}$ were different from those after treatment with CK. The effect of fertilizer application on the composition, relative abundance and community structure of dominant groups of soil bacteria was greater than that of straw mulching, which significantly reduced the soil bacterial richness, whereas straw mulching significantly increased the soil bacterial diversity.
\end{abstract}

Keywords: chemical fertilization, straw residue, high-throughput sequencing, Mollisol, bacterial composition

\section{Introduction}

Soil microorganisms are not only an important part of the soil, but are also the primary promoters of soil nutrient cycles. Changes in the soil microorganism community can reflect changing trends in soil quality to a certain extent (Zhang et al., 2005). Bacteria are the most abundant and widely distributed microbial community in plural, participating in nearly all biochemical cycles of terrestrial ecosystems, and playing an important role in maintaining the health of farmland ecosystems and soil productivity (Han et al., 2014). However, the research on soil bacteria is limited because most of them are uncultured due to the limitations of traditional molecular biological methods, such as denaturing gradient electrophoresis (DGGE) (Cao et al., 2013). In recent years, high-throughput sequencing technology has provided a powerful tool for the study of microbial molecular ecology, which has been widely applied in the study of environmental and soil microorganisms (Bartram et al., 2011). Chen et al. (2015) used Miseq high-throughput sequencing technology based on the Illumina platform to compare the soil bacterial community structure in different iron mining areas, which 
resulted in a deepened understanding of the mechanism by which heavy metal pollution influences the soil bacterial community structure and diversity. Fields such as those in one study (Tian et al., 2013) that were based on the Illumina platform of Miseq highthroughput sequencing technology, the leakage of $\mathrm{CO}_{2}$ sequestration scenario was studied to evaluate changes in the richness of the diversity and community structure of the farmland soil bacterial community; the results showed that changes occurred in the richness of the diversity and community structure of the cornfield soil bacterial community structure due to leakage of $\mathrm{CO}_{2}$ sequestration. The soil acid bacillus (Acidobacteria) bacteria showed a relative increase in May as a biological monitoring indicator of the effect of leakage of geologic storage of $\mathrm{CO}_{2}$ on the soil ecological system. High-throughput sequencing is simple, the results are stable, the repeatability is strong, and the consistency of the sequencing results is as high as $99.99 \%$; thus, this method allows the possibility for in-depth study of soil bacteria (Liu et al., 2014; Zhang et al., 2014).

Black soils (Mollisols) are a type of soil with characters of inherently fertile and productive. Around the world, there are four dominant Mollisol distribution areas, which are located in North America, in Russia and Ukraine, in South America and in north-eastern of China (Liu et al., 2019). Black soil is one of the important soils in the northeast plain of China and is primarily distributed in this area, covering an area of approximately 1.02 million square kilometres (Xing et al., 2005). The soil type is classed as a phaeozem (IUSS Working Group WRB, 2006). The black soils in Heilongjiang province are fertile, with relatively high cation exchange capacity, epipedon macroaggregate stability and high organic matter content (Hu et al., 2017). Crop stalks produced in the process of crop production are one of the important organic fertilizer sources. Simultaneously, crop stalks that are returned to the field as an important link in global organic agriculture have positive effects on maintaining farmland fertility, reducing fertilizer use and improving the land-soil carbon sink capacity (Zhang et al., 2013).

In recent years, returning straw to the field has been widely applied in agricultural production practice, and good results have been achieved with regard to fertilizing black soil and increasing crop yield (Wei et al., 2013; Zhu et al., 2014). Fertilization is one of the most profound agricultural measures affecting soil quality and its sustainable use. Different fertilization systems, soil microbial populations, quantities and activities lead to differences in soil biological fertility, and these differences affect the soil structure. Fertility and productivity have a profound effect (Wang et al., 2014).

Application of chemical and organic fertilizers has a certain effect on microorganism community structure and diversity in farmland soil. Wang et al. (2015) showed that the application of organic fertilizers could improve the diversity of soil fungi. Luo (2014) showed that long-term fertilization of brown soil significantly changed the soil microbial community structure and activity and significantly increased the diversity of bacteria and fungi in the brown soil. In a greenhouse simulation, Sapp et al. (2015) showed that fertilization had a certain effect on soil bacterial communities. Therefore, changes in the bacterial community structure, dominant populations and diversity in farmland soil after fertilization are important for maintaining soil fertility and the farmland ecological environment (Bao et al., 2000). Currently, the effects of fertilization and returning of straw to the field on microorganism groups in the uplands of black soil are rarely reported. Therefore, this study primarily adopted field experiments using the Miseq Illumina platform for high-throughput sequencing of soil 
bacterial $16 \mathrm{~S}$ rDNA to study the effect of different fertilization systems on the composition, structure and diversity of bacteria in black soil of an arid farmland ecosystem. The results will deepen the understanding of soil microbial groups and changes in soil microbial community structure, providing a theoretical basis for improving soil biological fertility of chernozems.

\section{Materials and Methods}

\section{Overview of the test site and test materials}

The trial began in 2012 at the Heilongjiang Academy of Agricultural Sciences in the Heilongjiang Province modern agriculture demonstration area $\left(45^{\circ} 49^{\prime} \mathrm{N}, 126^{\circ} 48^{\prime} \mathrm{E}\right.$, and altitude $117 \mathrm{~m}$ ) (Figure 1). The area has a mid-temperate continental monsoon climate with a short winter and summer, an annual average temperature of $4.5{ }^{\circ} \mathrm{C}$, an annual $\geq 10{ }^{\circ} \mathrm{C}$ effective accumulated temperature of over $2700{ }^{\circ} \mathrm{C}$, an annual crop growth period of approximately $155 \mathrm{~d}$, an annual average precipitation of $569.1 \mathrm{~mm}$, and precipitation primarily concentrated in June-September. The soil type is black soil, and soil nutrient contents in the $0-20 \mathrm{~cm}$ soil layer are as follows: organic matter of $29.56 \mathrm{~g} \cdot \mathrm{kg}^{-1}$, total nitrogen of $2.81 \mathrm{mg} \cdot \mathrm{kg}^{-1}$, available nitrogen of $79.56 \mathrm{mg} \cdot \mathrm{kg}^{-1}$, available phosphorus of $55.84 \mathrm{mg} \cdot \mathrm{kg}^{-1}$ and available potassium of $168.42 \mathrm{mg} \cdot \mathrm{kg}^{-1}$. The rice variety tested was long rice 21 .

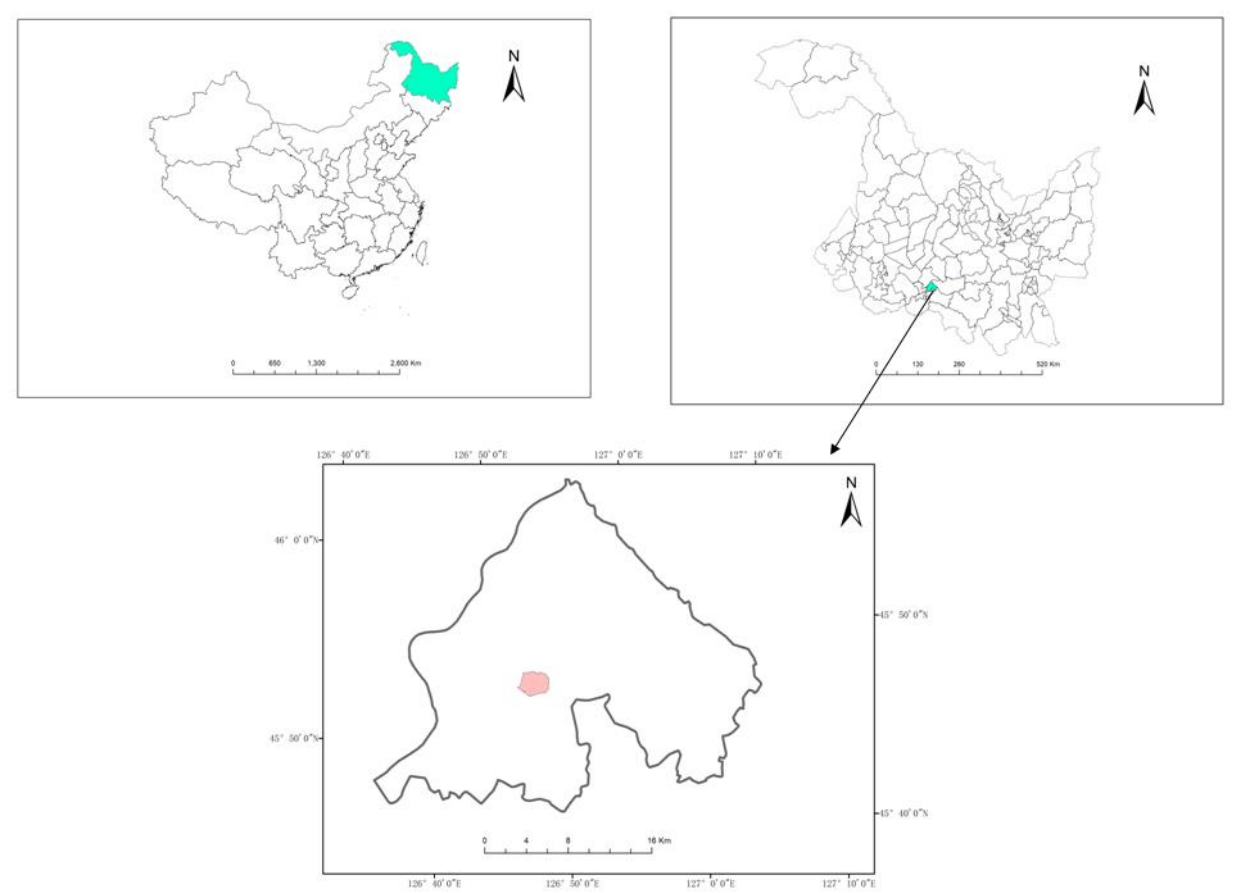

Figure 1. The research location of this experiment

\section{Experiment design}

The stubble crop before the experimental field was rice, and the planting mode was single-season rice, leaving the field free of crops in the winter. For the plots, the area was $24 \mathrm{~m}^{2}$. The straw used in the experiment was the rice straw harvested in the 
previous season, which was dried and cut into approximately $5 \mathrm{~cm}$ pieces and returned to the field evenly. To ensure the uniformity of the test, all the stubble straw was removed before the site was cleared and then returned to the field accurately according to the treatment. The crushed straw was spread evenly on the surface of the plot, mixed in the soil layer by rotary tiller, and the stubble field was transplanted to rice after a week.

Three treatments for the amount of straw returned to the field were used in the experiment, namely, no fertilizer or straw returned (the amount of straw was $0 \mathrm{~kg} / \mathrm{hm}^{2}$ ) to the field (CK), fertilizer with no straw returned (the amount of straw was $0 \mathrm{~kg} / \mathrm{hm}^{2}$ ) to field (F) and no fertilizer with straw returned (the amount of straw was $7500 \mathrm{~kg} / \mathrm{hm}^{2}$ ) to field (W). Each treatment was replicated 3 times in the field. All treatments were applied with the same amount of fertilizer (N3). Approximately $133 \mathrm{~kg} \cdot \mathrm{hm}^{-2}$ of nitrogen fertilizer (pure nitrogen) was applied, $46 \mathrm{~kg} \cdot \mathrm{hm}^{-2}$ of pure phosphorus was applied, and $75 \mathrm{~kg} \cdot \mathrm{hm}^{-2}$ of pure potassium was applied. Phosphate fertilizer was used as a base fertilizer $(100 \%)$ once. The fertilizer was applied to a base fertilizer $(50 \%)$, a turning green fertilizer $(30 \%)$ and a tillering fertilizer $(20 \%)$, and the amount of straw returned was $7500 \mathrm{~kg} / \mathrm{hm}^{2}$. Random permutations were used with 3 replicates. For the plots, the size was $24 \mathrm{~m}^{2}$. Seeding was performed on April 15; seedling planting was performed on May 15; and the seedling specifications were $30 \mathrm{~cm}$ (row spacing) x $13 \mathrm{~cm}$ (plant spacing) x 3 plants/hole. Other management measures were implemented uniformly according to the local conventional cultivation requirements. From 2012, we always continued these two treatments ( $\mathrm{N}$ and straw) every year in order to investigate the effects of long-term treatment on soil bacterial diversity and structure.

The arable layer soil (0-20 cm) was sampled in October 2017, with each processing district thoroughly incorporated with multipoint sampling. Each soil sample was divided into two parts. One hundred grams was returned to the lab in sterilized bags and stored at $-80{ }^{\circ} \mathrm{C}$ for the next step of molecular biology research. Approximately $800 \mathrm{~g}$ that remained was used to analyse the basic physical and chemical properties of the soil (Table 1); the basic physical and chemical properties of soil were determined with reference to the methods described by Bao et al. (2000).

Table 1. Chemical properties of the soil after different treatments

\begin{tabular}{c|c|c|c|c|c}
\hline Treatment & $\mathbf{p H}$ & $\begin{array}{c}\text { Organic matter } \\
(\mathbf{O C})(\mathbf{g} / \mathbf{k g})\end{array}$ & $\begin{array}{c}\text { Total N } \\
(\mathbf{g} / \mathbf{k g})\end{array}$ & $\begin{array}{c}\text { Available P }(\mathbf{A P}) \\
(\mathbf{m g} / \mathbf{k g})\end{array}$ & $\begin{array}{c}\text { Available K } \\
(\mathbf{A K}) \mathbf{( m g / k g})\end{array}$ \\
\hline CK & $7.09 \pm 0.02 \mathrm{a}$ & $16.01 \pm 0.15 \mathrm{~b}$ & $1.01 \pm 0.02 \mathrm{~b}$ & $8.02 \pm 1.02 \mathrm{~b}$ & $117.56 \pm 5.45 \mathrm{~b}$ \\
F & $5.47 \pm 0.03 \mathrm{c}$ & $17.05 \pm 0.20 \mathrm{a}$ & $1.22 \pm 0.03 \mathrm{a}$ & $26.08 \pm 1.56 \mathrm{a}$ & $145.64 \pm 6.52 \mathrm{a}$ \\
$\mathrm{W}$ & $6.25 \pm 0.01 \mathrm{~b}$ & $16.56 \pm 0.78 \mathrm{ab}$ & $1.08 \pm 0.01 \mathrm{~b}$ & $15.81 \pm 3.20 \mathrm{~b}$ & $137.65 \pm 6.52 \mathrm{ab}$ \\
\hline
\end{tabular}

Note: Different small letters in the same column indicate significant differences among treatments at a significance level of 0.05 . The same applies to the results described below. CK: no fertilizer or straw returned to the field; F: fertilizer with no straw returned; W: no fertilizer with straw returned

\section{Extraction of total DNA from soil microorganisms and high-throughput sequencing of bacteria}

A strong soil DNA extraction kit from the MOBIO Power Soil $®$ DNA Isolation Kit (USA) was adopted, and soil microbial genomic DNA was extracted according to the operation manual. A nucleic acid quantifier (NanoDrop ND-1000) was used detect the concentration and purity of the total DNA extracted from the soil. After the samples 
were purified, the DNA was sent to the MiSeq Illumina platform of Beijing Biomaker Company for sequencing.

Synthesizing specific primers with bar codes according to the designated sequencing area was the primary step of the high-throughput sequencing. PCR used TransGen AP221-02 and TransStart Fastpfu DNA Polymerase, with PCR conducted on an ABI GeneAmp ® 9700. All samples were conducted in accordance with the formal experimental conditions, and each sample was replicated 3 times. The PCR products of the same sample were mixed and detected by $2 \%$ agarose gel electrophoresis. PCR amplification was performed using primers 338F (5'-actcctacgggaggcagca-3') and 806R (5 '-ggactachvgggtwtctaat-3'), which amplified the V3-V4 region of 16S rDNA of soil bacteria (Zhang et al., 2015). The $20-\mu \mathrm{LPCR}$ reaction system was as follows: $4 \mu \mathrm{L}$ of $5 \times$ FastPfu Buffer, $2 \mu \mathrm{L}$ of $2.5 \mathrm{mmol} \cdot \mathrm{L}^{-1} \mathrm{dNTPs}, 0.4 \mu \mathrm{L}$ of forward primer $\left(5 \mu \mathrm{mol} \cdot \mathrm{L}^{-1}\right)$, $0.4 \mu \mathrm{L}$ of reverse primer $\left(5 \mu \mathrm{mol} \cdot \mathrm{L}^{-1}\right), 2 \mu \mathrm{L}$ of template (10 ng DNA), $0.4 \mu \mathrm{L}$ of FastPfu Polymerase (Trans Start Fastpfu DNA Polymerase, TransGen, China), and $10.8 \mu \mathrm{L}$ of ddH2O. PCR amplification procedure was performed as follows: $95{ }^{\circ} \mathrm{C}$ pre degeneration for $2 \mathrm{~min}$, modified at $55{ }^{\circ} \mathrm{C}$ and $95{ }^{\circ} \mathrm{C}$ for $30 \mathrm{~s}$ and $30 \mathrm{~s}$, annealing at $72{ }^{\circ} \mathrm{C}$, and extending for $30 \mathrm{~s}$, for a total of 30 cycles; completed at $72{ }^{\circ} \mathrm{C}$ and eventually extended to $10 \mathrm{~min}$. According to the results of the electrophoresis, preliminary quantitative PCR products were detected with the QuantiFluor ${ }^{\mathrm{TM}}$ - ST blue fluorescence quantitative system (Promega). Then, according to the volume of sequencing, each sample was quantified according to the corresponding percentage of hybrid.

\section{High-throughput sequencing data analysis}

The experiment used pair-end sequencing. First, quality control of raw data was conducted, and the sequences were connected with the Flash software (Magoč and Salzberg, 2011) to discard sequences that could not be connected. According to the test requirements, the bases below the mass value of the tail of the read were filtered with the window set to $50 \mathrm{bp}$. When the average mass value in the window was lower than $20 \mathrm{bp}$, the base at the back end from the window was cut off, and the reads below $50 \mathrm{bp}$ were filtered after quality control. According to the overlapping relationship between PE reads, the paired reads were spliced (merged) into a sequence, and the minimum overlap length was $10 \mathrm{bp}$. The maximum error ratio allowed in the overlapping region of the mosaic sequence was 0.2 , and the non-conformance sequence was screened. The box sequence at the end of the sequence was detected, and the minimum mismatch number was 0 . The sequence containing box at the beginning was inversely complemented, and the box was removed. The barcode on the test sequence was detected, and the samples were distinguished. The number of the barcode mismatches was 0 , the maximum primer mismatches was 2 , and the final sequence for analysis was obtained (Edgar et al., 2011).

With the application of QIIME (Quantitative Insights into Microbial Ecology), the sequences were classified into multiple OTUs (operational taxonomic units) according to similarities between the sequences. After OTU production, the OTUs contained in each sample and the number of sequences contained in each OTU was calculated. The uparse OTU (version 7.1; http://drive5.com/uparse/) method was used for clustering, with the OTU sequence similarity set at $97 \%$, to obtain representative OTU sequences (Edgar et al., 2011). Using uchime (version 4.2.40; http://drive5.com/usearch/manual/uchime_algo.html), PCR amplification of chimeric 
sequences was detected and removed from the OTUs (Quast et al., 2012). Using the usearch_global method (Edgar, 2010), the map of the optimized sequence was compared back to the OTU representative sequence, and the abundance statistics table of each sample sequence of an OTU was obtained. To obtain each OTU corresponding species classification information, the RDP classifier Bayesian algorithm at a $97 \%$ similarity level was used for analyses of the taxonomic OTU on behalf of the sequences, and at all levels (phylum, class, genus) for the statistical community composition of each sample, we specified the dominant population at the phylum, and class level relative abundance was greater than $10 \%$, with the relative abundance of the genus level greater than 1\%. The comparison database was as follows: Silva (Release115 http://www.arb-silva.de) (Chao et al., 1984). The OTUs with a similarity at $97 \%$ were selected to generate the expected dilution curve, and mothur was used to calculate the abundance indexes, Chaol (1984) and ACE (1993), and the diversity indexes, Shannon (Shannon, 1948a,b) and Simpson (Simpson, 1949). The bacterial community richness was represented by the Chaol and ACE indexes, and relatively high values indicated high community species richness. The Shannon index reflected the degree of diversity of a sample, and relatively high values indicated high diversity of community species. The Simpson index reflected the dominance of species.

\section{Statistical analysis}

Difference in soil physicochemical characteristics, Reads, bacterial alpha diversity indices of different treatments were compared using one-way analysis of variance, which was followed by the Duncan's difference test performed in IBM SPSS (version 17.0; Chicago, IL, USA) (Banerjee et al., 2016).

The shared and unique OTUs among different treatments were used to generate Venn diagrams using $\mathrm{R}$ software (version 3.3.2; $\mathrm{R}$ Development Core Team, 2017). Rarefaction curves were conducted by R software with "vegan" package based on OTU level (version 3.3.2; R Development Core Team, 2017). The heatmap representation of the top 50 classified genera in per treatment was built using the $\mathrm{R}$ software with "ggplot" and "pheatmap" packages (version 3.3.2; R Development Core Team, 2017). In order to find the relationships of soil physicochemical properties and soil bacterial community, Redundancy analysis (RDA) was used to analyze the soil physicochemical properties and soil bacterial community at OTU level. The RDA was performed by R software with "vegan" package.

\section{Results}

\section{Soil physicochemical properties}

As seen in Table 1, soil $\mathrm{pH}$ value ranged from 5.47 to 7.09 . Soil $\mathrm{pH}$ value under $\mathrm{F}$ treatment was the most acidic with 5.47 , followed by W treatment, while CK contained the highest soil $\mathrm{pH}$ value. There was significant difference among different treatment regarding soil $\mathrm{pH}, \mathrm{OC}, \mathrm{TN}, \mathrm{AP}$ and $\mathrm{AK}(P<0.05$, Table 1$)$. Interestingly, $\mathrm{OC}, \mathrm{TN}, \mathrm{AP}$ and AK exhibited highest value in the F treatment, with the values of $17.05 \mathrm{~g} / \mathrm{kg}$, $1.22 \mathrm{~g} / \mathrm{kg}, 26.08 \mathrm{mg} / \mathrm{kg}$ and $145.64 \mathrm{~g} / \mathrm{kg}$, respectively. 


\section{Sequencing results of soil samples and validation of sampling depth}

Using the bacterial 16S rDNA V3-V4 sequencing area, with low quality sequences filtered from the original sample, a total of 210,383 valid sequences and 183,232 readings (reads), clustering under 97\% similarity for OTUs for species classification, and the statistics of each sample for different OTU abundances were obtained; the nine samples produced 25,843 OTUs. As shown in Table 2, the soil treated with $\mathrm{W}$ contained the maximum number of OTUs, followed by the CK treatment, and the minimum number of OTUs occurred after treatment with F. Compared with the CK treatment, the number of soil OTUs decreased by $4.70 \%$ after treatment with F, whereas the number of soil OTUs increased by $11.9 \%$ after treatment with $\mathrm{W}$.

Table 2. Reads and OTU numbers of soil bacterial sequences after the different treatments

\begin{tabular}{c|c|c}
\hline Treatment & Reads & OTUs \\
\hline CK & $16889 \pm 5259 \mathrm{a}$ & $2655 \pm 172 \mathrm{ab}$ \\
F & $20539 \pm 6225 \mathrm{a}$ & $2530 \pm 95 \mathrm{~b}$ \\
W & $21447 \pm 4298 \mathrm{a}$ & $2973 \pm 46 \mathrm{a}$ \\
\hline
\end{tabular}

Different small letters in the same column indicate significant differences among treatments at a significance level of 0.05 . The same applies to the results described below. CK: no fertilizer or straw returned to the field; F: fertilizer with no straw returned; W: no fertilizer with straw returned

The rarefaction curve reflects the sample depth and can be used to evaluate whether the sequencing volume is sufficient to cover all groups. Figure 2 shows the dilution curve for all samples in this test under the condition of similarity of 0.97. As shown in Figure 2, all soil sample dilution curves tended to flatten, indicating that sampling was reasonable, and the confidence in the bacterial community structure in the actual environment was high, which could reflect the bacterial community of a soil sample in a relatively real way.

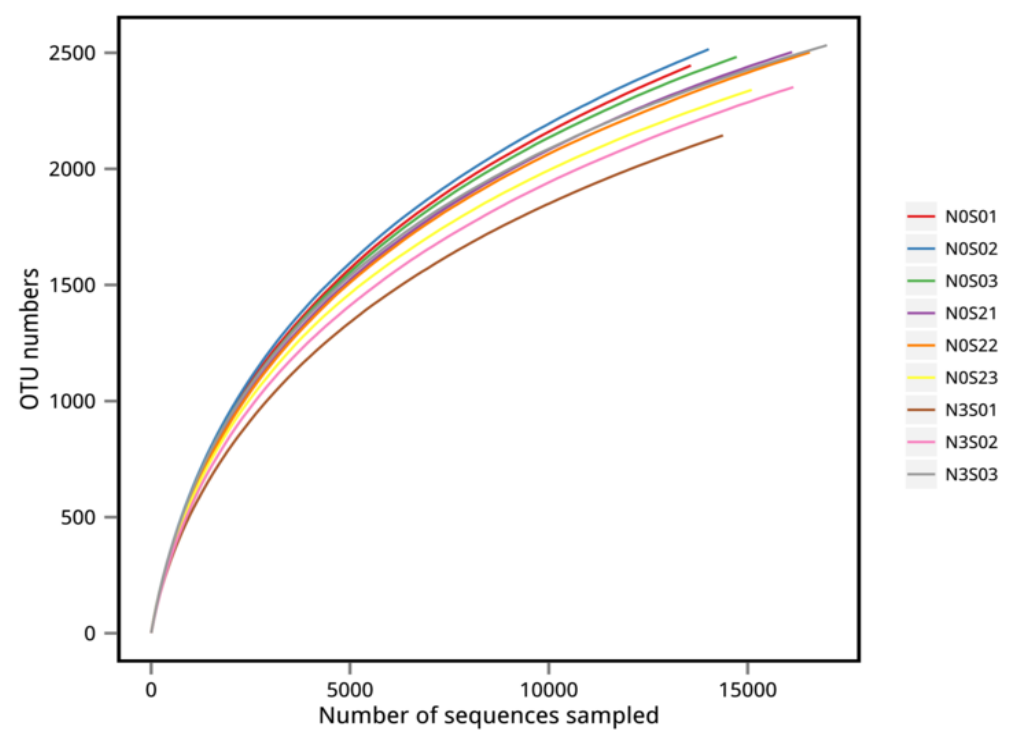

Figure 2. Rarefaction curves of OTUs clustered at $97 \%$ sequence identity across different soil samples. Note: NOSO represents CK; N3SO represents F; NOS2 represents W. CK: no fertilizer or straw returned to the field; $F$ : fertilizer with no straw returned; $W$ : no fertilizer with straw returned 


\section{Analysis of soil bacterial community richness and diversity}

From Table 3, the soil richness indexes (Chao1 and ACE indexes) of the F treatment were significantly lower than those of the $\mathrm{CK}$ and $\mathrm{W}$ treatments. No significant difference was detected between the bacterial richness indexes of the $\mathrm{W}$ and $\mathrm{CK}$ treatments. The ACE index of the F treatment was $27.4 \%$ lower than that of the CK treatment. The results showed that fertilization significantly reduced the richness of soil bacterial species, but straw returned to the field had little effect on the richness of soil bacterial populations. The Shannon index for soil bacteria treated with $F$ was significantly different from that for the bacteria treated with CK (Table 3), whereas the Shannon index for soil bacteria treated with $\mathrm{W}$ was significantly lower than that of those treated with CK (6.94), and the Shannon index for soil bacteria treated with W was $2.4 \%$ higher than that of those treated with CK. The results showed that the soil bacterial diversity was greatly improved, but fertilization had little effect on the soil bacterial diversity. The Simpson index of the F treatment was significantly higher than that of the CK and W treatments, and the Simpson index of the F treatment was $25.0 \%$ higher than that of the CK treatment, whereas the smallest Simpson index was obtained with the $\mathrm{W}$ treatment, which was significantly lower than that of the $\mathrm{CK}$ treatment by $25.0 \%$. The above results indicated that fertilization significantly reduced the soil bacterial richness and increased the soil bacterial dominance. Straw returned to the field improved the soil bacterial diversity and reduced the soil bacterial dominance.

Table 3. Richness and diversity indexes of soil bacteria after different treatments

\begin{tabular}{c|c|c|c|c}
\hline Treatment & ACE index & Chao1 index & Shannon index & Simpson index \\
\hline CK & $4468.75 \pm 72 \mathrm{a}$ & $4421.57 \pm 67 \mathrm{a}$ & $6.94 \pm 0.03 \mathrm{~b}$ & $0.004 \pm 0.0001 \mathrm{~b}$ \\
F & $3241.91 \pm 106 \mathrm{~b}$ & $4218.49 \pm 60 \mathrm{~b}$ & $6.92 \pm 0.04 \mathrm{~b}$ & $0.005 \pm 0.0001 \mathrm{a}$ \\
W & $4479.60 \pm 63 \mathrm{a}$ & $4330.81 \pm 52 \mathrm{a}$ & $7.11 \pm 0.12 \mathrm{a}$ & $0.003 \pm 0.0001 \mathrm{c}$ \\
\hline
\end{tabular}

Different small letters in the same column indicate significant differences among treatments at a significance level of 0.05 . CK: no fertilizer or straw returned to the field; F: fertilizer with no straw returned; W: no fertilizer with straw returned

\section{Analysis of soil bacterial groups}

At the phylum level, bacteria were distributed in 12 known phylum groups, in addition to 11 candidate phyla and an unclassified group. As shown in Figure 3, Proteobacteria, Acidobacteria, Bacteroidetes, Chloroflexi, Actinobacteria, Gemmatimonadetes, Nitrospirae, Verrucomicrobia, and Ingavibacteriae were detected. The relative abundance of a total of 11 bacterial phyla was greater, and the sum of the relative abundances accounted for more than $95 \%$ of the total amount of bacteria in the three treated soil samples.

The dominant bacterial groups in the $\mathrm{CK}$ and $\mathrm{F}$ treatments were Proteobacteria, Acidobacteria, Bacteroidetes, Chloroflexi and Actinobacteria. The return of straw reduced the relative abundances of Bacteroides and Actinobacteria, which decreased by 10.5 and $9.7 \%$, respectively. The relative abundances of Proteus and Acidophilus increased by 4.7 and $18.5 \%$, respectively. The composition and relative abundance of the dominant bacteria in soil treated with $\mathrm{W}$ were different from those in soil treated with $\mathrm{CK}$ and $\mathrm{F}$. The dominant bacteria in soil treated with $\mathrm{W}$ were deformed bacteria, Acidobacteria and Chloroflexi. Compared with treatment with CK, treatment with W 
increased the relative abundances of Proteobacteria, Acidobacteria and Chloroflexi by 7.5, 15.5 and 32.4\%, respectively. The relative abundance of Bacteroidetes decreased by $23.8 \%$.

W
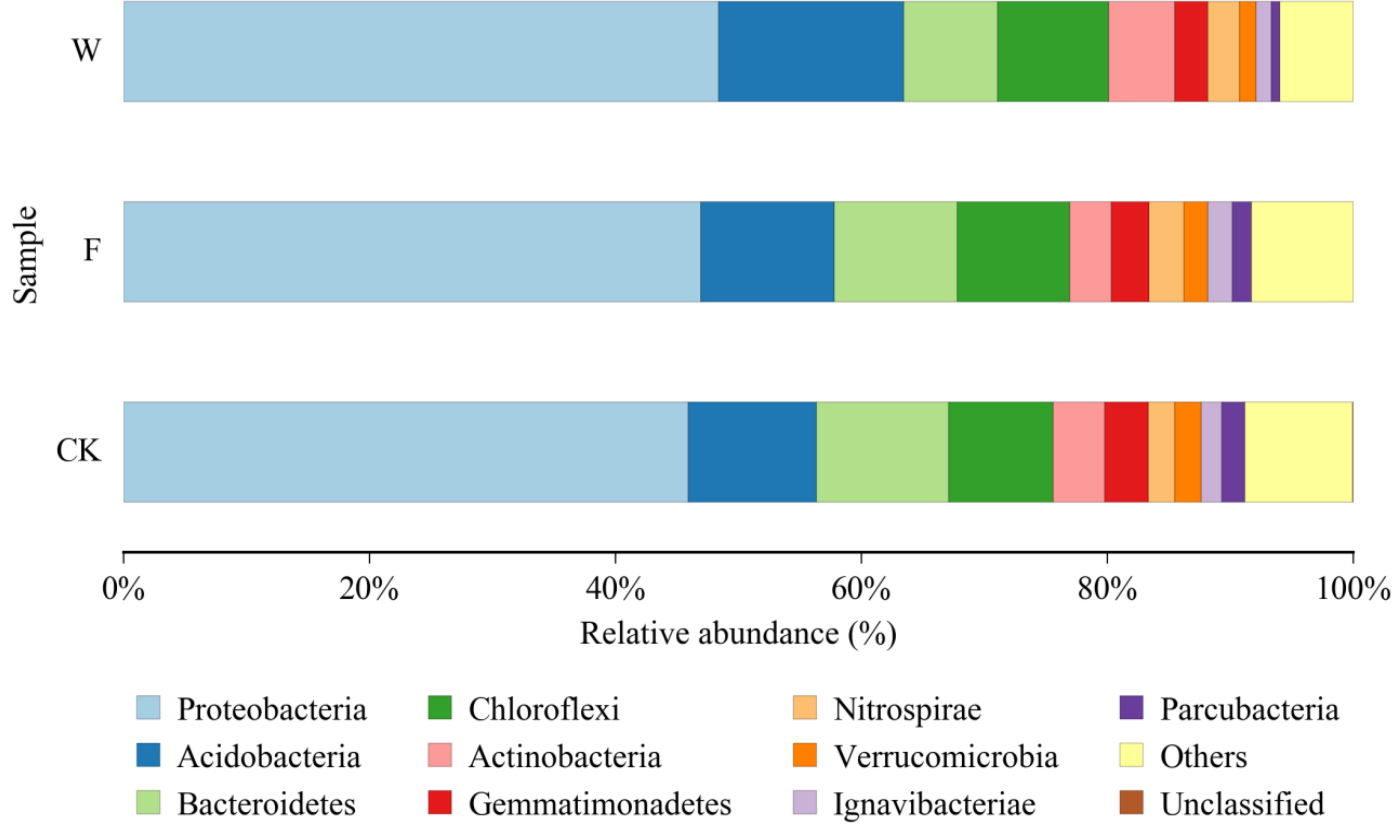

Figure 3. Comparison of bacterial groups at the phylum level after different treatments. CK: no fertilizer or straw returned to the field; F: fertilizer with no straw returned; W: no fertilizer with straw returned

At the class level, a total of 21 known classes of bacteria were obtained. In addition to unclassified classes, 40 candidate classes of bacteria were obtained. As shown in Figure 4, Betaproteobacteria, Deltaproteobacteria, Alphaproteobacteria, Gammaproteobacteria, Sphingobacteriia, Anaerolineace, Solibacteres, Nitrospira, Holophagea, Gemmatimonadetes, Actinobacteria, Cytophagia, Acidobacteria, Ignavibacteria, Blastocatellia, and the Bacteroidetes_vadinHA17 group were relatively abundant classes of bacteria, and the sum of the relative abundances accounted for more than $80 \%$ of the total amount of soil bacteria in the three treated soil samples.

Differences in the composition and relative abundance of the three dominant soil bacterial programmes were observed (Figure 4). The dominant bacteria in the soil treated with $\mathrm{CK}$ were $\alpha$-proteobacteria, $\beta$-proteobacteria and acid bacillus, and the dominant bacteria of the $\mathrm{F}$ treatment were $\alpha$-proteobacteria, sphingolipids, actinomycetes, and $\gamma$-proteobacteria.The dominant bacteria in the soil treated with $\mathrm{W}$ were $\alpha$-proteobacteria, $\beta$-proteobacteria, and sphingolipidae. Compared with the CK, fertilization increased the relative abundance of e. perciformis, actinomycetes, and sphingolibacilli by $19.2 \%, 46.4 \%$ and $45.3 \%$, respectively. The relative abundance of wild-deformation bacteria was reduced by $36.1 \%$. The relative abundance of bacillus costunica increased by $36.0 \%$, and the relative abundance of candidia-proteobacterium, extension-proteobacterium and acid-bacilli decreased by $5.8 \%, 18.8 \%$ and $28.1 \%$, respectively. 

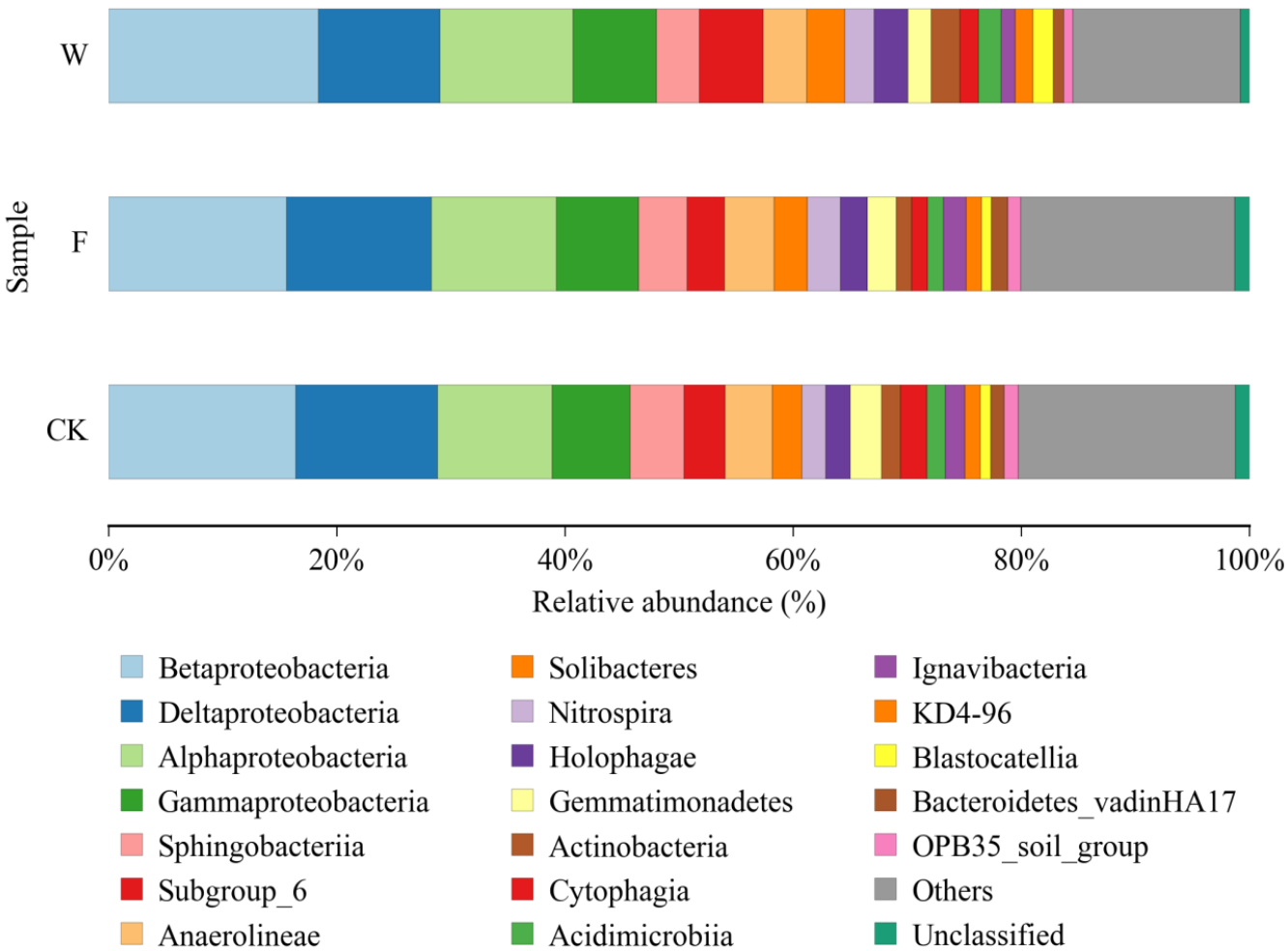

Figure 4. Comparison of bacteria groups at the class level after different treatments. CK: no fertilizer or straw returned to the field; F: fertilizer with no straw returned; W: no fertilizer with straw returned

A Venn diagram (Figure 5) was used to analyse the number and unique dominant genera (relative abundance $>1 \%$ ) shared by three fertilization-treated soil bacterial communities. The dominant genera with the most species (636) were in the F treatment group. In the CK treatment, 610 dominant genera were identified. Fertilizing and returning of straw to the field both significantly increased the number of dominant species in soil of which the dominant population in soil was the largest. The three samples had high relative abundance of Nitrosomonadaceae, Anaerolineaceae, and Gemmatimonas. The unique advantage of CK treatment was that 15 of the genera of rose bend occurred, and 24 genera had a unique advantage from $\mathrm{W}$ treatment. The maximum number of bacterial genera in the soil sample treated with $\mathrm{F}$ was 35 .

The relative abundance level of the top 50 bacteria was used to build a hierarchical clustering figure (hierarchical heatmap; Figure 6). The hierarchical clustering figure uses colour changes to visually define the size of the data values by colour depth, and the degree of the colour gradient reflects the similarities and differences in the data. As shown in Figure 6, the 9 soil samples were divided into two categories. The bacterial community structure of the 6 soil samples treated with $\mathrm{F}$ and $\mathrm{W}$ was similar, and the bacteria were clustered into one group. However, the bacteria from the 3 soil samples treated with CK were clustered into another group. The conclusion is that the effect of fertilization and straw return on the soil microbial community structure was significant at the genus level. The red part in the stratified cluster diagram shows the genus with the highest relative abundance, and as is clearly shown, the abundance of the primary soil bacterial communities under different treatments was different. 


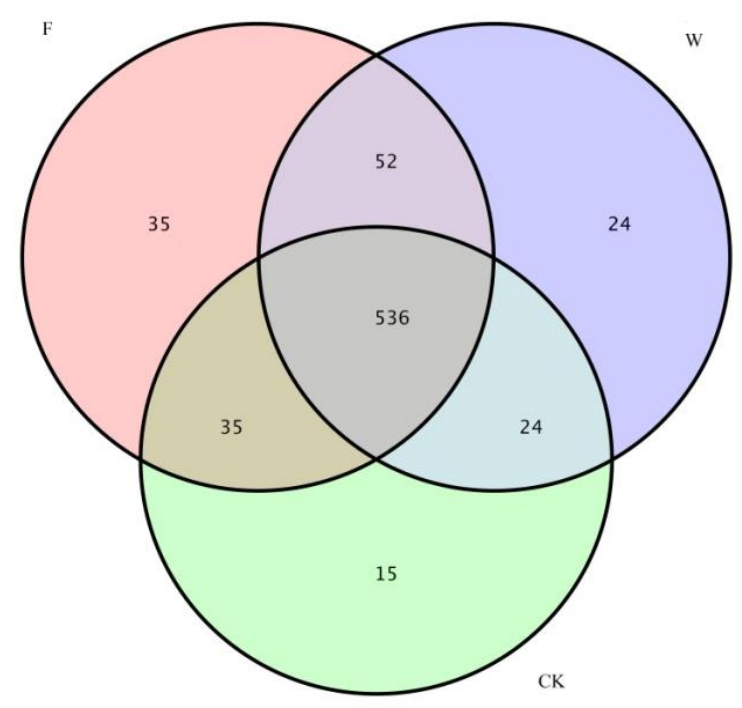

Figure 5. Venn diagram of the dominant genera among different soil treatments. CK: no fertilizer or straw returned to the field; F: fertilizer with no straw returned; W: no fertilizer with straw returned

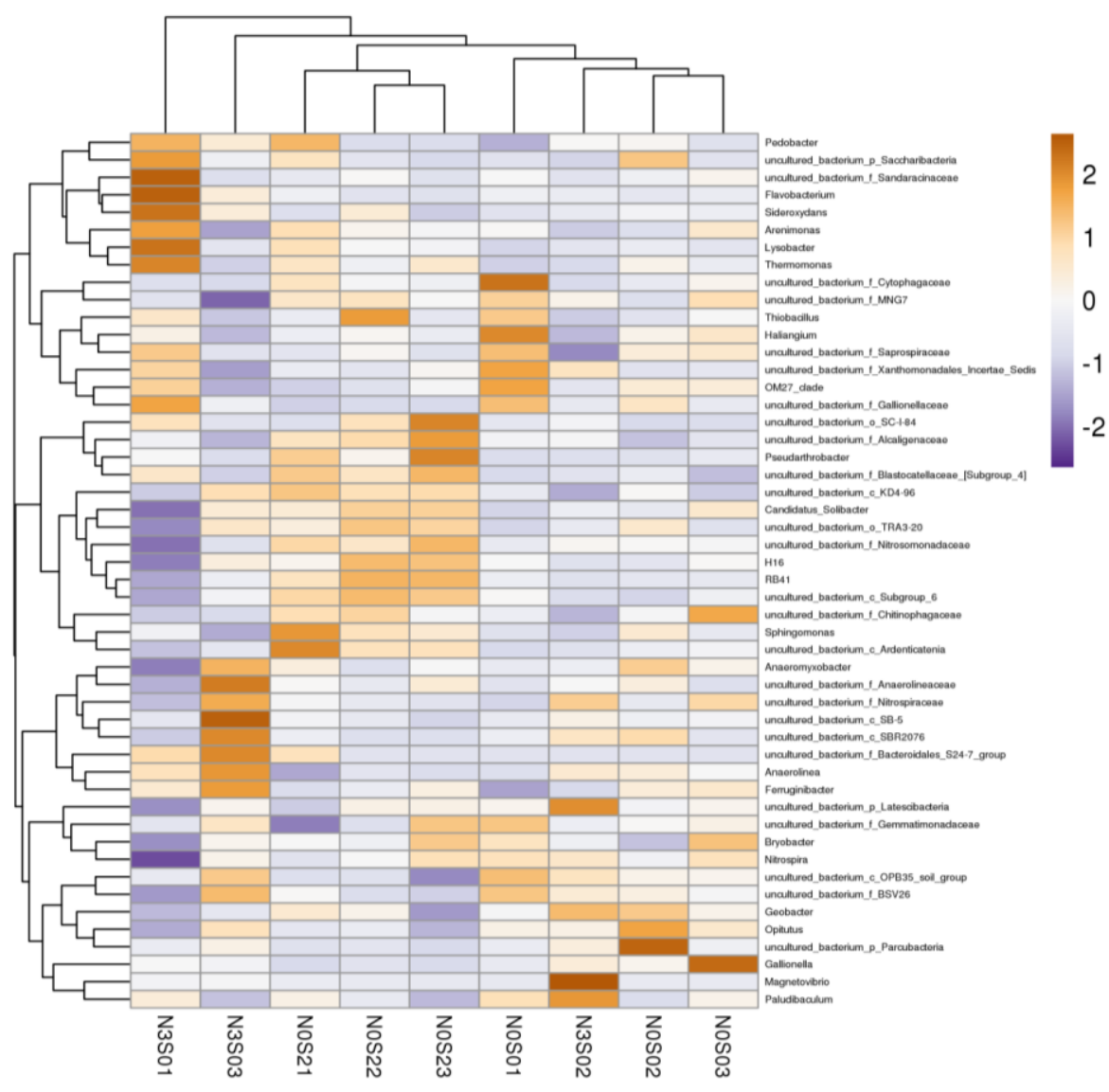

Figure 6. Hierarchical clustering diagram of bacteria at the genus level after different soil treatments. Note: NOSO represents $C K$; N3SO represents F; NOS2 represents W. CK: no fertilizer or straw returned to the field; F: fertilizer with no straw returned; W: no fertilizer with straw returned 


\section{Redundancy analysis of soil physicochemical properties and soil bacterial community}

RDA was used to evaluate the relationship of soil physicochemical properties and soil bacterial community (Figure 7). The first tow axes of the principal components analysis accounted for $34.03 \%$ of the total variance. The biplot showed a clear spatial separation among different soil samples. In fact, the axis 1 discriminated for the $\mathrm{W}$ treatment, while the axis 2 discriminated $\mathrm{F}$ treatment. The AP was situated in the first quadrant, soil $\mathrm{pH}$ in the second quadrant; soil $\mathrm{TN}$ in the third quadrant and $\mathrm{OC}$ in the fourth quadrant. The soil $\mathrm{pH}$ and $\mathrm{AP}$ was significant correlation with $\mathrm{F}$ treatment and the $\mathrm{OC}$ and $\mathrm{TN}$ was significant correlation with $\mathrm{W}$ treatment.

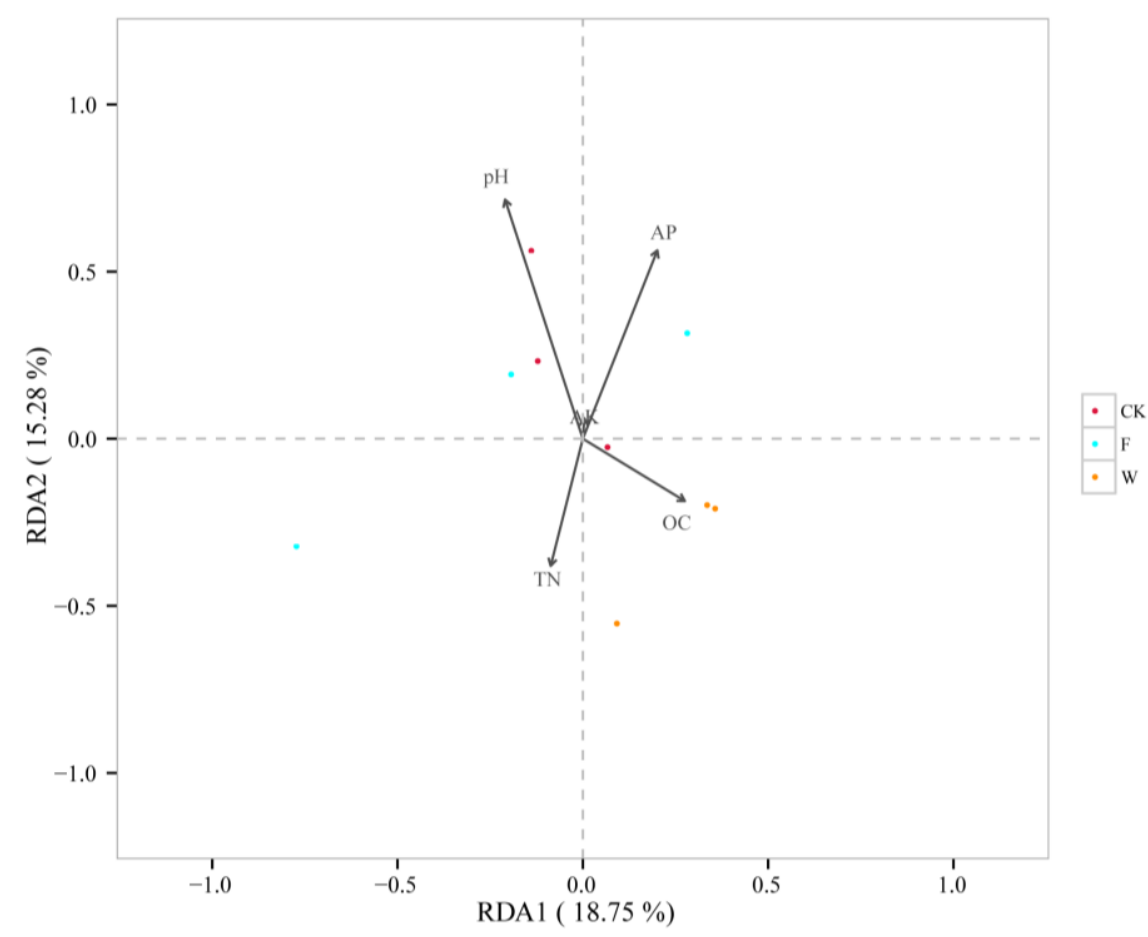

Figure 7. Redundancy analysis of soil physicochemical properties and soil bacterial community. Note: NOSO represents CK; N3SO represents F; NOS2 represents W. CK: no fertilizer or straw returned to the field; F: fertilizer with no straw returned; W: no fertilizer with straw returned

\section{Discussion}

This study used Illumina platform Miseq high-throughput sequencing technology to conduct amplification sequencing of $16 \mathrm{~S}$ rDNA V3-V4 of soil bacteria, and the good coverage index of each sample shows that under the condition of a similarity of 0.97 , OTUs covered more than $99 \%$ of the bacteria in the soil. Simultaneously, sequencing also identified many unclassified bacteria, indicating that high-throughput sequencing can obtain comprehensive biological information and also identify some unrecognized and classified bacteria. However, the relative abundance of the unclassified bacteria in the soil among the 3 treatments did not differ significantly at each taxonomic level.

Soil microbial diversity is relatively stable, and short-term fertilization has no significant effect on soil microorganisms (Shi et al., 2010), but long-term reasonable fertilization can have a certain effect on soil microbial community structure and diversity (Rousk et al., 2010; Liu et al., 2014) showed that the physical and chemical 
properties of soil, particularly the soil $\mathrm{pH}$ and organic matter, had significant effects on the soil bacterial community composition and diversity.

In this paper, straw returned to the field significantly improved soil bacterial diversity. The straw returned to the field not only increased the soil organic matter content in the process of decomposition but may also provide rich nutrition for microbes in the straw products produced during the process of decay, such as sugars, eggs, endoplasmic reticulum, amino acids, vitamins, organic acids, and phenols, among others (Sapp et al., 2015). Simultaneously, straw returned to the field can improve soil structure and increase soil permeability, thereby increasing microbial species and soil bacterial diversity. Fertilizer application significantly reduced the richness of soil bacteria and led to a significant decrease in soil $\mathrm{pH}$ and soil consolidation, which was not conducive to the growth of aerobic bacteria.

The relative abundance of dominant bacterial groups in soil has a certain correlation with the physical and chemical properties of soil (Wang et al., 2015). In this study, the relative abundance of acid-bacilli was negatively correlated with total nitrogen and available potassium in soil. The relative abundance of Bacteroides was positively correlated with total nitrogen, available potassium and available phosphorus in soil. The relative abundance of SPP was positively correlated with soil $\mathrm{pH}$ and negatively correlated with organic matter, total nitrogen and phosphorus. The relative abundances of ore-deformis and campus-deformis were positively correlated with $\mathrm{pH}$ and negatively correlated with the contents of organic matter, total nitrogen and available phosphorus. The relative abundance of probiotics was negatively correlated with $\mathrm{pH}$ and positively correlated with the contents of organic matter, total nitrogen and available phosphorus. The relative abundance of Nitrospira was significantly positively correlated with $\mathrm{pH}$ and negatively correlated with organic matter, total nitrogen and available phosphorus (Lauber et al., 2008).

In the black soil of northeast China, bacterial groups were abundant, and the dominant bacteria were Proteobacteria, Actinomycetes, and Bacteroides, and the relative abundance of Proteobacteria was the largest (36.5-45.2\%). Bacterial classes at an advantage were beta bacteria, phylum $\Delta$-deformation, alpha deformation phylum, and gamma, with beta deformation bacteria being the largest phylum based on relative abundance. These results are consistent with those of (Liu et al., 2014) regarding bacteria in black soil in northeast China and with those of Sapp et al. (2015). The application of chemical fertilizer and the return of straw to the field changed the physical and chemical properties of the soil. The relative abundance of Proteobacteria in black soil in northeast China is less than that in the three treated soil groups. As the largest group of bacteria, many groups can perform nitrogen fixation and adapt to various complex environments (Liu et al., 2014). Therefore, changes in environmental conditions have little effect on their distribution and relative abundance. Acidophilus bacteria are widely distributed in soil and sediment and are acidophilic. However, in this paper, the lowest values of soil $\mathrm{pH}$ and relative abundance of Acidobacteria occurred after fertilization, confirming results of (Liu et al., 2014) that the growth of Acidobacteria in soil was not only affected by soil $\mathrm{pH}$ but also by other factors such as soil organic matter and crop growth status. Actinomycetes can promote the decomposition of animal and plant residues in soil, play an important role in the decomposition process of crop stalks after they are returned to the field, and play a certain role in the natural nitrogen cycle (Lauber et al., 2008). Fertilization treatment and straw returned to the field significantly increased the relative abundance of 
Actinomycetes in the soil, which might be related to the increase in total nitrogen content after treatments with fertilization and straw returned to the field. Fertilization and straw return to the field also had certain effects on some functional bacteria related to elemental transformation. For example, Sphingomonas is a new type of biological resource that is primarily involved in the decomposition of aromatic compounds. Compared with the CK treatment, straw return to the field significantly reduced the relative abundance of Sphingomonas. Fertilization and straw return to the field also had a certain effect on the relative abundance of bacteria associated with biological nitrogen fixation. Fertilization and straw return to the field significantly reduced the relative abundance of Nitrospira, which led to a large increase in the relative abundance of Rhizobium. Fertilization significantly reduced the relative abundance of Gemmatimonas associated with phosphorus metabolism.

The effect of fertilization on the composition, relative abundance and community structure of dominant groups of soil bacteria was greater than that of straw returned to the field. The mechanisms by which fertilization and straw return to the field affect the community structure of black soil bacteria in northeast China require further research.

\section{Conclusions}

The long-term effects of fertilizer application on the composition, relative abundance and community structure of dominant groups of soil bacteria were greater than those of straw returned to the field. Additionally, fertilizer application significantly reduced soil bacterial richness, whereas straw returned to the field significantly increased soil bacterial diversity.

Our results found that long-term fertilizer application was the main factor that resulted the soil bacterial community changed in black soil. This provided a guidance to explore environmentally friendly agricultural fertilization measures in the future: while increasing soil fertility and increasing crop yields, as the same time measures should also be taken to maintain a suitable $\mathrm{pH}$ of the soil, so as to achieve equal emphasis on agricultural production and ecological benefits. Of course, this study only analyzed the changes of the soil bacterial community. For special functional groups (such as nitrogen-fixing bacteria, nitrifying bacteria, etc.) and other microbial species (such as archaea, fungi, and miniature animals) that play an important role in the soil ecosystem did not carried out. The systematic study of various types of microorganisms in the soil ecosystem will be an important aspect of our future research. This will be of great help for us to understand the function of the soil ecosystem and the relationship between the soil microbial community and soil fertility and crop yield. Furthermore, it provides a solid foundation for us to explore environmentally friendly and sustainable agriculture.

Acknowledgement. National key R\&D plan (2016YFD0300204); Heilongjiang Youth Fund Project (QC2018026) as well as Heilongjiang Academy of Agricultural Sciences-Agricultural Science and Technology Innovation Project (HNK2019CX1302).

\section{REFERENCES}

[1] Banerjee, S., Kirkby, C. A., Schmutter, D., Bissett, A., Kirkegaard, J. A., Richardson, A. E. (2016): Network analysis reveals functional redundancy and keystone taxa amongst 
bacterial and fungal communities during organic matter decomposition in an arable soil. Soil Biology \& Biochemistry 97: 188-198.

[2] Bao, S. D. (2000): Soil and Agricultural Chemistry Analysis. - Beijing: China Agriculture Press.

[3] Bartram, A. K., Lynch, M. D. J., Stearns, J. C. (2011): Generation of multimillionsequence 16S rRNA gene libraries from complex microbial communities by assembling paired-end Illumina reads. - Appl. Environ. Microbiol 77: 3846-3852.

[4] Cao, W. J., Sui, X., Han, S. J. (2013): Molecular biology technology and its application in soil microbial diversity. - Bioprocess 3: 23-28.

[5] Chao, A. (1984): Non-Parametric Estimation of the Number of Classes in a Population. Scandinavian Journal of Statistics 11: 265-270.

[6] Chao, A., Yang, M. C. K. (1993): Stopping rules and estimation for recapture debugging with unequal failure rates. - Biometrika 80: 193-201.

[7] Chen, H., Si, Y. X., Xing, Y., Li, Y. (2015): Illumina MiSeq sequencing investigation on the contrasting soil bacterial community structures in different iron mining areas. Environ Sci Pollut 22: 10788-10799.

[8] Edgar, R. C. (2010): Search and clustering orders of magnitude faster than BLAST. Bioinformatics 26: 2460-2461.

[9] Edgar, R. C., Haas, B. J., Clemente, J. C., Quince, C., Knight, R. (2011): UCHIME improves sensitivity and speed of chimera detection. - Bioinformatics 27: 2194-2200.

[10] Han, Y. F., Yi, W. H., Wang, W. B. (2014): Soil bacteria diversity in continuous cropping poplar plantation by high throughput sequencing. - J Shandong Univ. Nat Sci 49: 1-6.

[11] Hu, X. J., Liu, J. L., Wei, D., Zhu, P., Cui, X. A., Zhou, B. K., Chen, X. L., Jin, J., Liu, X. B. (2017): Effects of over 30-year of different fertilization regimes on fungal community compositions in the black soils of Northeast China. - Agriculture Ecosystems \& Environment 248: 113-122.

[12] IUSS Working Group WRB. (2006): World reference base for soil resources 2006 (2 $2^{\text {nd }}$ ed.). - World Soil Resources Report No. 103. FAO Rome.

[13] Lauber, C. L., Strickland, M. S., Bradford, M. A., Fierer, N. (2008): The influence of soil properties on the structure of bacterial and fungal communities across land-use types. Soil Bio Biochem 40: 2407-2415.

[14] Liu, J. J., Sui, Y. Y., Yu, Z. H., Shi, Y., Chu, H. Y., Jin, J., Liu, X. B., Wang, G. H. (2014): High throughput sequencing analysis of biogeographical distribution of bacterial communities in the black soils of northeast China. - Soil Biol Biochem 70: 113-122.

[15] Liu, J. J., Yao, Q., Li, Y. S., Zhang, W., Wang, G. H. (2019): Continuous cropping of soybean alters the bulk and rhizospheric soil fungal communities in a mollisol of Northeast China. - Land Degradation \& Development, DOI: 10.1002/ldr.3378.

[16] Luo, P. Y. (2014): Effect of long-term fertilization on microbial community in a brown soil under crop-rotation system. - MS thesis. Shenyang: Shenyang Agricultural University.

[17] Magoč, T., Salzberg, S. L. (2011): FLASH: fast length adjustment of short reads to improve genome assemblies. - Bioinformatics 27: 2957-2963.

[18] Pan, J. L., Dai, W. A., Shang, Z. H., Guo, R. Y. (2013): Review of research progress on the influence and mechanism of field straw residue incorporation on soil organic matter and nitrogen availability. - Chin J Eco-Agricul 21: 526-535.

[19] Quast, C., Pruesse, E., Yilmaz, P., Gerken, J., Schweer, T., Yarza, P., Peplies, J., Glöckner, F. O. (2012): The SILVA ribosomal RNA gene database project: Improved data processing and web-based tools. - Nucleic Acids Research 41: 590-596.

[20] Rousk, J., Bååth, E., Brookes, P. C., Lauber, C. L., Lozupone, C., Caporaso, J. G., Fierer, N., Knight, R. (2010): Soil bacterial and fungal communities across a $\mathrm{pH}$ gradient in an arable soil. - International Society for Microbial Ecology 4: 1340-1351.

[21] Sapp, M., Harrison, M., Hany, U., Charlton, A., Thwaites, R. (2015): Comparing the effect of digestate and chemical fertiliser on soil bacteria. - Appl Soil Ecol 86: 1-9. 
[22] Shannon, C. E. (1948a): A mathematical theory of communication. - The Bell System Technical Journal 27: 379-423.

[23] Shannon, C. E. (1948b): A mathematical theory of communication. - The Bell System Technical Journal 27: 623-656.

[24] Shi, P., Gao, Q., Wang, S. P., Zhang, Y. (2010): Effects of continuous cropping of corn and fertilization on soil microbial community functional diversity. - Acta Ecol Sini 30: 6173-6182.

[25] Simpson, E. H. (1949): Measurement of Diversity. - Nature 163: 688.

[26] Tian, D., Ma, X., Li, Y. E., Zha, L. S., Wu, Y., Zou, X. X., Liu, S. (2013): Research on soil bacteria under the impact of sealed $\mathrm{CO} 2$ leakage by high-throughput sequencing technology. - Environ Sci 34: 4096-4104.

[27] Wang, Y., Li, J., Cao, Z. P., Yang, H. F. (2014): Effects of long-term fertilization on soil fungi. - Chin J Eco-Agricul 22: 1267-1273.

[28] Wang, F. W. (2015): The effects of fertilization and straw residue incorporation on bacterial communities in lime concretion black soil. - MS thesis. Hefei: Anhui Agricultural University.

[29] Wei, W., Xu, Y. L., Zhu, L., Han, X. Z., Li, S. (2013): Effect of long-term fertilization on soil microbial communities in farmland of black soil. - Acta Pedologica Sinica 50: 372380.

[30] Xing, B. S., Liu, X. B., Liu, J. D., Han, X. Z. (2005): Physical and chemical characteristics of a typical Mollisol in China. - Communications in Soil Science \& Plant Analysis 35(13-14): 1829-1838.

[31] Zhang, W., Wei, H. L., Gao, H. W. (2005): Advances of studies on soil microbial diversity and environmental impact factors. - Chin J Ecol 24: 48-52.

[32] Zhang, B., Pang, C. Q., Qin, J. T., Liu, K. L., Xu, H., Li, H. X. (2013): Rice straw incorporation in winter with fertilizer-N application improves soil fertility and reduces global warming potential from a double rice paddy field. - Biology and Fertility of Soils 49: 1039-1052.

[33] Zhang, Y. G., Cong, J., Lu, H., Yang, C., Yang, Y., Zhou, J., Li, D. (2014): An integrated study to analyze soil microbial community structure and metabolic potential in two forest types. - PLoS One 9: e93773.

[34] Zhang, Y., Wang, X., Hu, M., Li, P. (2015): Effect of hydraulic retention time (HRT) on the biodegradation of trichloroethylene wastewater and anaerobic bacterial community in the UASB reactor. - Appl Microbiol Biotechnol 99: 1977-1987.

[35] Zhu, L. Q., Hu, N. J., Yang, M. F., Zhan, X., Zhang, Z. (2014): Effects of different tillage and straw return on soil organic carbon in a rice-wheat rotation system. - PLoS One 9: e88900. 